\title{
Depth-Dependent Contrast in Probability-Current Imaging from Channeling in Crystalline Materials
}

\author{
Zhen Chen ${ }^{1}$, Kayla X. Nguyen ${ }^{1}$, Celesta S. Chang ${ }^{1}$, Michael C. Cao ${ }^{1}$, David A. Muller ${ }^{1,2}$ \\ 1. School of Applied and Engineering Physics, Cornell University, Ithaca NY 14853, USA \\ 2. Kavli Institute at Cornell for Nanoscale Science, Ithaca NY 14853, USA
}

Differential phase contrast (DPC) imaging is a useful technique for studying magnetic or electric field distributions in materials at both the nanoscale and atomic resolution [1,2]. A new generation of fast high dynamic range pixel array detectors provides the possibility of obtaining the whole electron diffraction pattern at each probe position in scanning transmission electron microscopy (STEM) [3]. These 4D-STEM datasets enable quantitative and momentum-resolved DPC, or center of mass (CoM), imaging which potentially provides more physical information about the specimen compared to DPC images from traditional segmented detectors. Furthermore, CoM images can be understood as the quantum mechanical probability current flow [4] and give direct measurements of the electron beam's lateral propagation within the specimen. Within the strong phase object approximation, which is only valid if the real-space probe shape is unchanged by scattering in the specimen, CoM images can be expressed as the gradient of the specimen potential [5,6]. Here, we demonstrated a probability current flow oscillation within a crystal beyond the strong phase object approximation [7], using $\mathrm{SrTiO}_{3}$ as an example.

Fig. 1 shows experimental CoM images at low magnification (a) and atomic resolution (b). CoM image using a 4.3 mrad upper cut-off angle on the diffraction pattern, the left panel of Fig. 1 (a), shows clear thickness fringes. Extending the cut-off angle to $120 \mathrm{mrad}$, greatly reduces the visibility of the thickness fringes (right panel of Fig. 1 (a)). Crystal tilt produces strong contrast, especially in the large cut-off angle CoM images, marked by the black dashed box on the right panel of Fig. 1 (a). Fig. 1 (b) shows the contrast reversal of CoM images varying either cut-off angle or specimen thickness. For a $12 \mathrm{~nm}$ thick specimen, the CoM signal at the Ti site reverses contrast from negative-positive for 11 mrad cut-off angle to positivenegative for 120 mrad cut-off angle, emphasized by red dashed boxes on Fig. 1 (b). Another contrast reversal at the Ti site can also be seen in the $11 \mathrm{mrad}$ cut-off angle CoM images by changing the specimen thickness from $10 \mathrm{~nm}$ to $40 \mathrm{~nm}$.

Simulation provides insight into these oscillations. Figs. 2 (a) and (b) show line profiles of the CoM signal with different cut-off angles using frozen phonon simulations along $\mathrm{Sr}-\mathrm{Sr}$ and Ti-O directions within the $\mathrm{SrTiO}_{3}$ unit cell. CoM images using 4.3 mrad cut-off angle in Fig. 2 (a) show a thickness oscillation with a period of about $660 \AA$ for both $\mathrm{Sr}$ and $\mathrm{TiO}$ columns, and there is another finer oscillation with a period of about $110 \AA$ of CoM from large collection angle in Fig. 2 (b). An intuitive physical picture of the oscillation can be obtained from the real space probe propagation (Fig. 2 (c)) showing when the electrons channel back and forth, there must be a probability current flows to redistribute probe intensity. A similar period of oscillation with a larger absolute CoM intensity from multi-slice simulations excluding thermal diffuse scattering reveals that elastic scattering dominates the oscillation. A simple model considering only the interference of 1s-state and free-space propagation [8] can predict well the finer thickness oscillation in large cut-off angle CoM but not the low cut-off angle oscillation. This suggests that more delocalized Bloch states should be considered for low cut-off angle CoM images. Furthermore, larger convergence-angle probes will excite a broader range of states making the CoM oscillation more complicated and less coherent, leading to more less pronounced oscillations. [9] 
References:

[1] N Shibata, et al, Nat. Phys. 8 (2012) p. 611.

[2] M Lohr, et al, Ultramicroscopy 117 (2012), p.7.

[3] MW Tate, et al, Microscopy and Microanalysis 22 (2016), p. 237.

[4] A Lubk, J Zweck, Phys. Rev. A 91 (2015), 023805.

[5] R Close, et al, Ultramicroscopy 159 (2015), p. 124.

[6] I Lazić, et al, Ultramicroscopy 160 (2016), p. 265.

[7] CS Chang, et al, Microscopy and Microanalysis 22 (2016), p. 1414.

[8] SD Findlay et al, Ultramicroscopy 110 (2010) p. 903.

[9] Research supported by PARADIM, an NSF MIP (DMR-1539918), with electron microscope facilities support from CCMR, an NSF MRSEC (DMR-1120296).

(a) $4.3 \mathrm{mrad}$

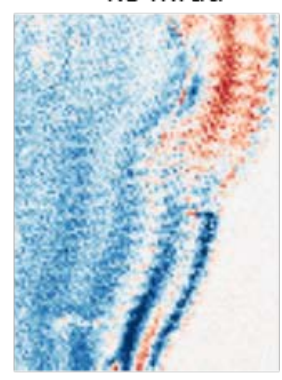

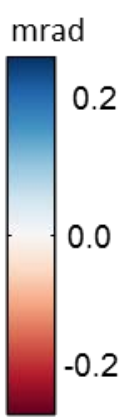

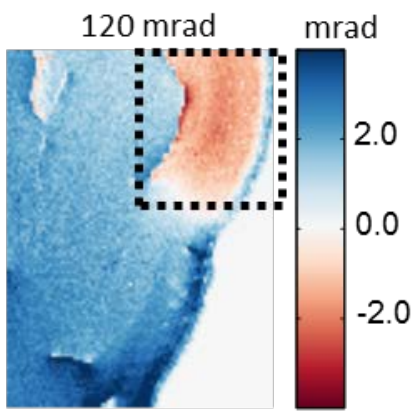

(b)

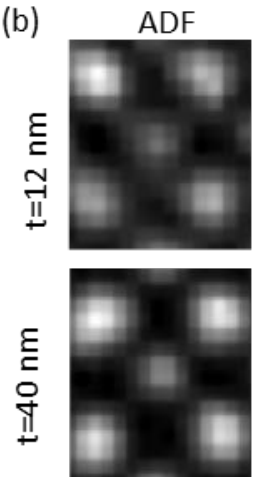

$11 \mathrm{mrad}$

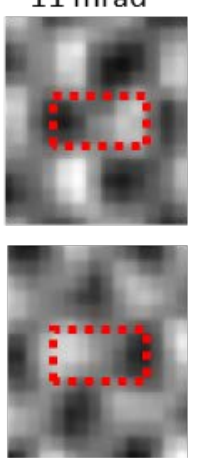

$120 \mathrm{mrad}$

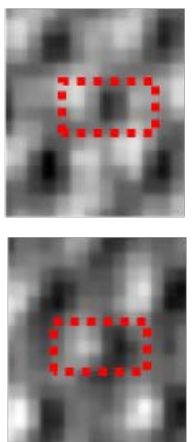

Figure 1. Experimental center of mass (CoM) images, $\left\langle\mathrm{p}_{\mathrm{x}}\right\rangle$. (a) Low magnification CoM images from $\mathrm{SrTiO}_{3}$ using a 10 mrad probe-forming semi-angle at $200 \mathrm{kV}$; (b) Atomic resolution CoM images with simultaneous ADF reference images for two different sample thicknesses using a 30 mrad probe-forming semi-angle at $120 \mathrm{kV}$. The upper cut-off angle of diffraction patterns used to form the CoM images is labeled on top of each image.
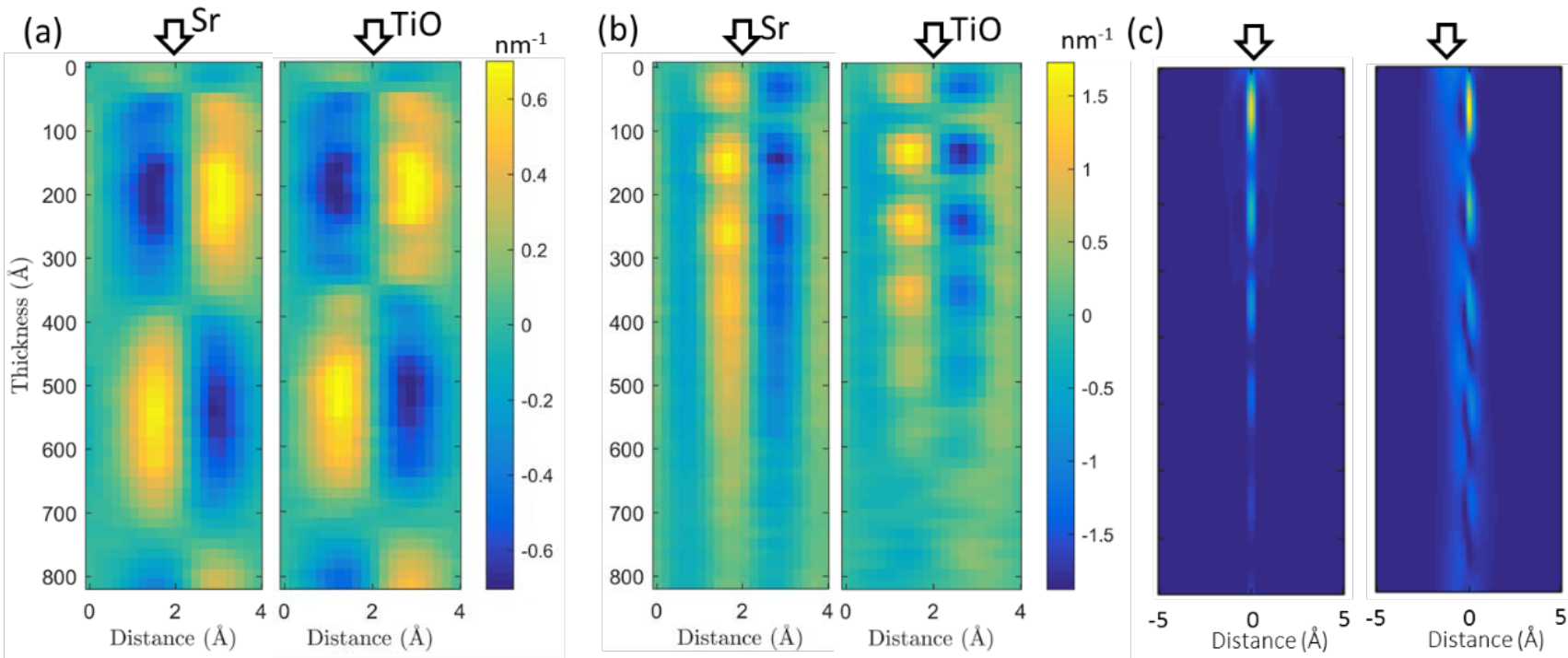

Figure 2. Simulated CoM images showing thickness oscillations in the probability current flow along $\mathrm{Sr}$ and $\mathrm{TiO}$ columns in [001] $\mathrm{SrTiO}_{3}$ using a 10 mrad probe-forming semi-angle at $200 \mathrm{kV}$. CoM line profiles are for cut-off angles of (a) $4.3 \mathrm{mrad}$ and (b) $120 \mathrm{mrad}$. (c) Real-space beam propagation for a probe directly on (left) and $0.8 \AA$ away from (right) Ti column. 